\title{
Gaudibilidad y percepción de estado de salud en una muestra mexicana
}

\author{
Joel Omar González-Cantero; Víctor Hugo González-Becerra; José Guadalupe Salazar-Estrada
}

How to cite this article:

González-Cantero, J. O., González-Becerra, V. H., \& Salazar-Estrada, J. G. (2019). Gaudiebility and health status perception in a Mexican sample. Acta Colombiana de Psicología, 22(2), 13-20. doi: http://www.doi.org/10.14718/ACP.2019.22.2.2

Recibido, abril 20/2018; Concepto de evaluación, agosto 3/2018; Aceptado, septiembre 18/2018

\author{
Joel Omar González-Cantero* \\ Universidad de Guadalajara, Guadalajara, México \\ ORCID: https://orcid.org/0000-0003-2158-2903 \\ Víctor Hugo González-Becerra \\ Universidad de Guadalajara, Guadalajara, México \\ ORCID: https://orcid.org/0000-0001-7744-6543 \\ José Guadalupe Salazar-Estrada \\ Universidad de Guadalajara, Guadalajara, México \\ ORCID: https://orcid.org/0000-0002-2199-1275
}

\begin{abstract}
Resumen
En la actualidad, para el abordaje del proceso salud-enfermedad se requiere, además de controlar la enfermedad, preservar en lo posible la calidad de vida de la persona. En este sentido, el objetivo del presente trabajo fue identificar la relación entre la gaudibilidad y la percepción del estado de salud en una muestra de mexicanos, y comparar dichas variables según el género de los participantes. Para esto, se utilizó un diseño transversal y correlacional con la aplicación de la Escala de Gaudibilidad y la versión corta del Cuestionario de Salud $(S F-36)$. En total, participaron 285 personas de entre 14 y 78 años $(M=32.13$, $D E=13.38$ ), reclutados por medio de la técnica de bola de nieve, con quienes se formó una muestra no probabilística. Los resultados indicaron una relación estadísticamente significativa, positiva y moderada entre la gaudibilidad y la salud general de los participantes $(\rho=.407, p<.001)$, debido a que las personas con alta gaudibilidad percibieron menos riesgos en salud general en comparación con las personas con baja y moderada gaudibilidad $(R M=4.527,2.434-8.419)$. Asimismo, se identificó una diferencia estadísticamente significativa en cuanto al género en los factores funcionamiento físico $(\mathrm{z}=-2.293$, $\mathrm{p}=.022)$ y salud mental $(z=-2.243, p=.025)$ del $S F-36$, ya que los hombres refirieron un nivel mayor en ambos casos. Se concluye que la gaudibilidad tiene una influencia relevante sobre la percepción del estado de salud.

Palabras clave: gaudibilidad, estado de salud, calidad de vida, psicología de la salud, psicología positiva, mexicanos.
\end{abstract}

\section{Gaudiebility and health status perception in a Mexican sample}

\begin{abstract}
The current approach to the health-illness process requires, in addition to disease control, the preservation of the person's quality of life. This study has the purpose of identifying the relationship between gaudiebility and the perception of health status in a Mexican sample, as well as comparing these variables by gender. A cross-sectional and correlational study was conducted for such purposes. Gaudiebility was measured with the Gaudiebility Scale and the perception of health status with the MOS 36Item Short-Form Health Survey (SF-36). The sample consisted of 285 people between the ages of 14 and $78(M=32.13, S D=$ 13.38), who were recruited with the snowball sampling method, forming a non-probability sample. Results indicate a statistically significant positive and moderate relationship between gaudiebility and the General Health of people $(\rho=.433, p<.001)$. Thus, people with high gaudiebility perceive less risks to their General Health compared with people who have low and moderate gaudiebility $(R M=4.527,2.434-8.419)$. Furthermore, a statistically significant difference was identified by gender regarding Physical Functioning $(z=-2.293, p=.022)$ and Mental Health $(z=-2.243, p=.025)$ on the $S F-36$, where men reported a higher level in both cases. It was concluded that gaudiebility has a relevant influence on the perception of health status.

Key words: Gaudiebility, health status, quality of life, health psychology, positive psychology, Mexicans.
\end{abstract}

\footnotetext{
Centro de Investigación en Comportamiento y Salud, Centro Universitario de los Valles, Carretera Guadalajara-Ameca, Km 45.5, C. P. 46600, Ameca, Jalisco, México. Tel.: +52 (375) 7580500, ext.47342.joel.gonzalez@academicos.udg.mx
} 


\title{
Gaudibilidade e percepçáo do estado de saúde em uma amostra mexicana
}

\begin{abstract}
Resumo
$\mathrm{Na}$ atualidade, para a abordagem do processo saúde-doença requer-se, além de controlar a doença, preservar, na medida do possível, a qualidade de vida da pessoa. Nesse sentido, o objetivo do presente trabalho foi identificar a relação entre a gaudibilidade e a percepção do estado de saúde em uma amostra de mexicanos e comparar tais variáveis de acordo com o gênero dos participantes. Para isso, utilizou-se um desenho transversal e correlacional com a aplicação da Escala de Gaudibilidade e a versão curta do Questionário de Saúde ( $S F-36$ ). No total, participaram 285 pessoas com idades entre 14 e 78 anos $(M=32.13, D P=13.38)$ recrutados por meio da técnica de bola de neve, com quem se formou uma amostra não probabilística. Os resultados indicaram uma relação estatisticamente significativa, positiva e moderada entre a gaudibilidade e a saúde geral dos participantes $(\rho=.407, p<.001)$, devido ao fato de que as pessoas com alta gaudibilidade perceberam menos riscos em saúde geral em comparação com as pessoas com gaudibilidade baixa e moderada $(R M=4.527,2.434$ - 8.419). Igualmente, identificou-se uma diferença estatisticamente significativa com respeito ao gênero nos fatores funcionamento físico $(z=-2.293, p=.022)$ e saúde mental $(z=-2.243, p=.025)$ do $S F-36$, já que os homens indicaram um maior nível em ambos os casos. Conclui-se que a gaudibilidade tem uma influência relevante sobre a percepção do estado de saúde.

Palavras-chave: gaudibilidade, estado de saúde, qualidade de vida, psicologia da saúde, psicologia positiva, mexicanos
\end{abstract}

\section{Introducción}

La tasa de esperanza de vida en México en el año 2016 fue de 75.2 años (Instituto Nacional de Estadística y Geografía, 2018), una tasa mucho más alta en comparación con la de décadas anteriores. Sin embargo, no se puede decir que el aumento de esta conlleve consigo el aumento de la calidad de vida, y esto puede explicarse por el riesgo de presentar en edades más avanzadas enfermedades crónico-degenerativas no transmisibles que implican tener que lidiar con complicaciones progresivas que terminan afectando el bienestar subjetivo y las condiciones de salud de las personas.

En la actualidad, para los sistemas sanitarios es un desafío no solo proporcionar tratamientos efectivos para el control de las enfermedades, sino también integrales y que garanticen la calidad de vida de las personas, y por esto es necesario que los profesionales involucrados en el estudio del proceso de salud-enfermedad atiendan de manera adecuada las demandas de bienestar psicológico de la población.

Teniendo esto en cuenta, se necesita de una participación más activa de profesionales de la salud-como es el caso de los psicólogos- frente a la prevención y promoción de la salud con modelos de atención a problemáticas de salud no solo orientados a la obtención de efectos inmediatos, sino que tengan un mayor énfasis en atender los factores que disminuyen la calidad de vida relacionada con la salud de las personas. Tal es el caso de la psicología positiva, donde se puede encontrar un gran número de estudios que revelan la importancia de las variables psicológicas moduladoras de la calidad de vida.
Particularmente, la psicología positiva ha mostrado un interés especial en el estudio del hedonismo -que puede aumentar mientras más se experimenta (Veenhoven, 2003)en relación con la identificación de factores asociados al afrontamiento de problemas relacionados con el proceso de salud-enfermedad. Y es en este contexto donde surge el concepto de gaudibilidad, cuyos antecedentes se remontan a la antigua Grecia, en donde el llamado "hedonista" se enfocaba en la búsqueda y estudio del placer, así como en la supresión del dolor como objetivo o razón de ser.

Un antecedente más reciente de la gaudibilidad es el concepto de "refuerzo de sensibilidad", según el cual los individuos con alta sensibilidad de refuerzo muestran mayor interés, persistencia y perseverancia hacia ciertos estímulos o situaciones que quienes no encuentran dicho refuerzo (Pickering \& Corr, 2008; Pickering, Corr \& Gray, 1999). No obstante, a diferencia del "refuerzo de sensibilidad", la gaudibilidad tiene en cuenta moduladores tanto cognitivos -como la imaginación, la concentración, el sentido del humor, creencias y valoraciones que conducen al disfrute- como emocionales -que conducen a experimentar sensaciones gratificantes 0 , incluso, empoderamiento- y conductuales -que abarcan aspectos gratificantes, actividades y retos(Sánchez-Teruel \& Robles-Bello, 2017).

Asimismo, la gaudibilidad implica un conjunto de procedimientos que sirven para modificar el control inadecuado de mecanismos implicados en procesos como la evasión, con el fin de orientarlos hacia una relación más natural y de disfrute con respecto a los acontecimientos que puedan mejorar el bienestar subjetivo (Montgomery, 2009). En este sentido, Padrós y Fernández-Castro (2008) definen la gaudibilidad como: 
un constructo que engloba todos aquellos procesos que median entre los estímulos y el disfrute que las personas experimentan, es decir, el conjunto de moduladores que regulan las sensaciones subjetivas de vivir experiencias gratificantes en mayor o menor grado de intensidad, en mayor o menor número de situaciones y durante periodos de tiempo más o menos prolongado(s). De manera que cuando más alta es la gaudibilidad, más probabilidades tienen las personas de disfrutar (p. 414).

Ahora bien, podría decirse que el desarrollo de un nivel alto de gaudibilidad está relacionado con factores de diversa índole, como los económicos, biológicos o demográficos, pero, como señalan Sánchez-Teruel y Robles-Bello (2017), los factores que modulan la gaudibilidad en mayor medida son los psicológicos.

En la literatura científica, las investigaciones sobre gaudibilidad se han enfocado en evaluar su relación con algunos trastornos y problemas psicológicos, como el estrés postraumático (Koenen, Stellman, Sommer \& Stellman, 2008), algunas adicciones, problemas sexuales (Mimiaga et al., 2012), trastornos obsesivo-compulsivos (Macy et al., 2013) y depresión mayor (Padrós, Martínez-Medina \& Graff, 2014), por lo cual se considera conveniente identificar su relación con variables relacionadas con el proceso de salud-enfermedad.

Con base en lo anterior, se observa que la gaudibilidad todavía no tiene el suficiente desarrollo teórico ni empírico que evidencie su relación con la calidad de vida y la salud de las personas (Jose, Lim \& Bryant 2012; Padrós, 2002; Padrós \& Fernández, 2001; Sprangers et al., 2010), lo cual justifica el objetivo del presente estudio: identificar la relación entre la gaudibilidad y la percepción del estado de salud de una muestra de mexicanos de la región de los Valles, Jalisco, y comparar ambas variables según el género de los participantes.

\section{Método}

\section{Tipo de estudio}

Se utilizó un diseño transversal y correlacional.

\section{Participantes}

El procedimiento para reclutar a los participantes fue el de bola de nieve, con el cual se conformó una muestra no probabilística, en donde los criterios de inclusión fueron: (a) ser mexicano, (b) saber leer y escribir y (c) residir en la zona de los Valles de Jalisco. En total, se incluyeron 285 participantes de entre 14 y 78 años $(M=32.13, D E=13.38)$, de los cuales el $36.5 \%$ eran hombres y el $63.5 \%$, mujeres; el $50.9 \%$ reportó ser soltero, el $42.8 \%$, casado, el $2.5 \%$, viudo, el $0.7 \%$, divorciado, y el $3.2 \%$ refirió otro estado civil; el $40.3 \%$ tenía educación primaria y secundaria, el $36.8 \%$, preparatoria, el $21.1 \%$, licenciatura, y el $1.8 \%$ maestría o doctorado; el $59.3 \%$ contaba con empleo; y el $40.1 \%$ reportó vivir en zona rural, mientras que el $59.9 \%$, en una zona urbana.

\section{Instrumentos}

Cuestionario de datos sociodemográficos. Se elaboró un cuestionario específicamente para este estudio con el fin de recolectar información sociodemográfica de los participantes, como en lo referente a la edad, el estado civil, el nivel de escolaridad, la ocupación y la zona de residencia.

Escala de Gaudibilidad. Se utilizó la Escala de Gaudibilidad creada por Padrós y Fernández-Castro (2008), compuesta por 23 ítems con cinco opciones de respuesta, que van de "completamente en desacuerdo" (0) a "completamente de acuerdo" (4). El puntaje total se obtiene de la suma de los 23 ítems, que puede oscilar entre 0 y 92 , donde entre más alto sea el puntaje, mayor se considera el nivel de gaudibilidad. Los reactivos 15,19 y 22 se califican de manera inversa.

Como señalan Padrós-Blázquez, Herrera-Guzmán y Gudayol-Ferré (2012), se ha demostrado evidencia satisfactoria de la confiabilidad de esta escala en México tanto a partir del procedimiento test-retest, $(r=.723, p<.001)$ como por medio del análisis del alfa de Cronbach $(\alpha=.820)$.

Debido a que el puntaje total de esta escala se puede agrupar en niveles, para este estudio los puntajes entre 0 y 74 corresponden a una gaudibilidad baja y moderada, mientras que aquellos de 75 o más se catalogaron como gaudibilidad alta. En este trabajo, la Escala de Gaudibilidad obtuvo una confiabilidad adecuada, con un alfa de Cronbach de .856 .

Cuestionario de Salud SF-36. Este cuestionario, creado por Ware y Sherbourne (1992), evalúa la calidad de vida relacionada con la salud y, por ende, proporciona un perfil del estado de salud. Específicamente, consta de 36 preguntas distribuidas en ocho factores, a saber: funcionamiento fisico (FF), rol físico (RF), dolor corporal (DC), salud general (SG), vitalidad (V), funcionamiento social (FS), rol emocional (RE) y salud mental (SM).

Con respecto a su calificación, entre mayor sea el puntaje obtenido en el cuestionario, mejor es el estado de salud que percibe la persona. Para cada factor, los ítems son codificados, agregados y transformados en una escala 
con un rango de 0 (el peor estado de salud) a 100 (el mejor estado de salud). Este cuestionario obtuvo un coeficiente de confiabilidad alfa de Cronbach de .93 en población clínica mexicana (Martínez-Hernández, Segura-Méndez, AntonioOcampo, Torres-Salazar \& Murillo-Gómez, 2010); y este coeficiente, en población no clínica, ha oscilado entre .56 y .84 para los factores que lo conforman (Zúñiga, CarrilloJiménez, Fos, Gandek \& Medina-Moreno, 1999). Este instrumento permite la comparación entre poblaciones de distintas áreas geográficas, en distintos periodos de tiempo y en diferentes sistemas sanitarios, por lo cual es muy utilizado por la Secretaría de Salud de México.

En este estudio, el Cuestionario de Salud SF-36 obtuvo un alfa de Cronbach adecuada en general (.907) y en cada uno de los factores: funcionamiento fisico $(\alpha=.870)$, rol físico $(\alpha=.908)$, dolor corporal $(\alpha=.825)$, salud general $(\alpha=.723)$, vitalidad $(\alpha=.342)$, funcionamiento social $(\alpha=.676)$, rol emocional $(\alpha=.828)$, salud mental $(\alpha=.813)$.

\section{Procedimiento}

La recolección de datos la realizó -en una sesión de entre 20 y 25 minutos- un grupo de cinco estudiantes de psicología entrenados en una sesión de cuatro horas, en la cual se analizaron las variables principales de la investigación, así como el procedimiento para la aplicación de los instrumentos psicométricos. La aplicación de los instrumentos fue presencial y de autorreporte en lugares que cumplían con las condiciones de iluminación, ventilación e infraestructura adecuadas para la tarea. En la aplicación, los aplicadores dieron una breve explicación del propósito de la investigación y a quienes aceptaron se les pidió que leyeran y, en caso de estar de acuerdo, firmaran el consentimiento informado.

\section{Análisis estadístico}

El análisis estadístico se realizó con el programa informático IBM SPSS Statistics, v. 24. Inicialmente, se utilizó el alfa de Cronbach para evaluar la confiabilidad de la Escala de Gaudibilidad y el Cuestionario de Salud $S F-36$ en el presente grupo de participantes; posteriormente, se realizó la prueba de normalidad para las variables gaudibilidad y estado de salud con base en la prueba de
Kolmogorov-Smirnov -debido a que la muestra es mayor a 50 participantes-; $y$, teniendo en cuenta los resultados de la prueba de normalidad, y con el fin de evaluar la relación entre gaudibilidad y la percepción del estado de salud, se realizaron dos análisis: una correlación de Spearman (adecuada para asociar dos variables cuando la muestra no tiene una distribución normal) y la razón de momios para medir el riesgo. Adicionalmente, se utilizó la $U$ de MannWhitney para comparar las dos variables según el género de los participantes. Por último, se calculó el tamaño del efecto con la $\mathrm{r}$ de Rosenthal en dichas comparaciones.

\section{Consideraciones éticas}

Las personas que aceptaron participar en el estudio lo manifestaron en la carta de consentimiento informado, donde se especificó que la presente consistía en una investigación de riesgo mínimo. Este consentimiento se basó en la Declaración de Helsinki y la Ley General de Salud de los Estados Unidos Mexicanos en su capítulo quinto "Investigación para la salud", artículo 100; y siguió los preceptos del Código Ético del Psicólogo que emite la Sociedad Mexicana de Psicología.

\section{Resultados}

Los resultados muestran una correlación estadísticamente significativa, pero débil, entre la gaudibilidad y todos los factores del estado de salud (véase Tabla 1). Sin embargo, el coeficiente de correlación más alto entre variables fue el de la gaudibilidad con la salud general, el cual consiste en la valoración personal del estado de salud -que incluye la situación actual y las perspectivas futuras- y la resistencia a enfermar.

Con respecto al análisis para identificar el riesgo que representa la baja y moderada gaudibilidad en comparación con una alta gaudibilidad sobre la percepción del estado de salud, se encontró que solamente el factor salud general estaría afectado, es decir, que las personas con alta gaudibilidad perciben menos riesgos en salud, en general, en comparación con las personas que tienen baja y moderada

Tabla 1.

Correlación de la gaudibilidad con los factores de la percepción del estado de salud

\begin{tabular}{|c|c|c|c|c|c|c|c|c|}
\hline & $\begin{array}{l}\text { Funcionamiento } \\
\text { físico }\end{array}$ & Rol físico & $\begin{array}{l}\text { Dolor } \\
\text { corporal }\end{array}$ & $\begin{array}{l}\text { Salud } \\
\text { general }\end{array}$ & Vitalidad & $\begin{array}{l}\text { Funcionamiento } \\
\text { social }\end{array}$ & $\begin{array}{l}\text { Rol } \\
\text { emocional }\end{array}$ & $\begin{array}{l}\text { Salud } \\
\text { mental }\end{array}$ \\
\hline Spearman & $.155^{* *}$ & $.222 * * *$ & $.200 * *$ & $.407 * * *$ & $.246^{* * *}$ & $.231 * * *$ & $.128 *$ & $.229 * * *$ \\
\hline
\end{tabular}

Nota. En el caso del dolor corporal, la correlación es positiva porque, de acuerdo con el $S F-36$, un mayor puntaje se codifica como ausencia de este factor. $* * * p<.001 ; * * p<.01 ; * p<.05$. 
Tabla 2.

Riesgo de percepción de un estado de salud negativo según el nivel de gaudibilidad

\begin{tabular}{|c|c|c|c|}
\hline Factor & $\begin{array}{c}\text { Gaudibilidad } \\
\text { baja y moderada } \\
(N=231) \\
\%(n)\end{array}$ & $\begin{array}{c}\text { Gaudibilidad } \\
\text { alta } \\
(N=54) \\
\%(n)\end{array}$ & $\begin{array}{c}R M \\
95 \% \mathrm{IC}\end{array}$ \\
\hline Funcionamiento físico & $12.6(29)$ & $9.3(5)$ & $\begin{array}{c}1.407 \\
(0.518-3.821)\end{array}$ \\
\hline Rol físico & $14.7(34)$ & $7.4(4)$ & $\begin{array}{c}2.157 \\
(0.732-6.362)\end{array}$ \\
\hline Dolor corporal & $34.6(80)$ & $25.9(14)$ & $\begin{array}{c}1.514 \\
(0.777-2.947)\end{array}$ \\
\hline Salud general & $77.1(178)$ & $57.4(23)$ & $\begin{array}{c}4.527 \\
(2.434-8.419)\end{array}$ \\
\hline Vitalidad & $78.8(182)$ & $70.4(38)$ & $\begin{array}{c}1.564 \\
(0.805-3.037)\end{array}$ \\
\hline Funcionamiento social & $28.1(65)$ & $16.7(9)$ & $\begin{array}{c}1.958 \\
(0.906-4.233)\end{array}$ \\
\hline Rol emocional & $34.6(80)$ & $33.3(18)$ & $\begin{array}{c}1.060 \\
(0.566-1.984)\end{array}$ \\
\hline Salud mental & $43.3(100)$ & $31.5(17)$ & $\begin{array}{c}1.661 \\
(0.884-3.121) \\
\end{array}$ \\
\hline
\end{tabular}

Nota. $R M=$ razón de momios; $\mathrm{IC}$ = intervalo de confianza.

gaudibilidad. Sin embargo, con respecto a los demás factores del estado de salud, no se encontraron relaciones significativas (véase Tabla 2).
Por último, con respecto a la comparación por género de la gaudibilidad y la percepción del estado de salud, las únicas diferencias estadísticamente significativas se observaron

Tabla 3.

Comparación de las medias obtenidas en la Escala de Gaudibilidad y el Cuestionario de Salud SF-36

\begin{tabular}{|c|c|c|c|c|c|}
\hline & $\begin{array}{l}\text { Hombres } \\
(n=104)\end{array}$ & $\begin{array}{l}\text { Mujeres } \\
(n=181)\end{array}$ & $\begin{array}{c}\text { Total } \\
(n=285)\end{array}$ & \multirow{2}{*}{$z$ de Mann-Whitney $(p)$} & \multirow{2}{*}{ r de Rosenthal } \\
\hline & $M(D E)$ & $M(D E)$ & $M(D E)$ & & \\
\hline Gaudibilidad & $64.08(12.70)$ & $63.70(11.21)$ & $63.84(11.76)$ & $-.007(p=.994)$ & -0.000 \\
\hline $\begin{array}{l}\text { Funcionamiento } \\
\text { físico }\end{array}$ & 92.35 (13.93) & $89.00(15.00)$ & $90.22(14.68)$ & $-2.293(p=.022)$ & -0.135 \\
\hline Rol físico & 89.67 (26.64) & $86.18(30.77)$ & $87.46(29.33)$ & $-.984(p=.325)$ & -0.058 \\
\hline Dolor corporal & $80.81(20.69)$ & $76.96(21.44)$ & $78.36(21.22)$ & $-1.657(p=.098)$ & -0.098 \\
\hline Salud general & $65.14(16.91)$ & $61.76(18.14)$ & $63.00(17.74)$ & $-1.294(p=.196)$ & -0.076 \\
\hline Vitalidad & $61.65(14.23)$ & $58.21(15.59)$ & $59.47(15.17)$ & $-1.783(p=.075)$ & -0.105 \\
\hline $\begin{array}{l}\text { Funcionamiento } \\
\text { social }\end{array}$ & $82.45(18.88)$ & $78.93(19.91)$ & $80.21(19.58)$ & $-1.473(p=.141)$ & -0.087 \\
\hline Rol emocional & $78.52(33.78)$ & $73.50(38.87)$ & $75.33(37.12)$ & $-.830(p=.407)$ & -0.049 \\
\hline Salud mental & $77.96(14.86)$ & $73.82(16.07)$ & $75.33(15.74)$ & $-2.243(p=.025)$ & -0.132 \\
\hline
\end{tabular}

Nota. $\mathrm{r}$ de Rosenthal = tamaño del efecto. 
en los factores funcionamiento fisico y salud mental, con un mayor nivel en los hombres, aunque en ambos casos el tamaño del efecto es muy pequeño (véase Tabla 3 ).

\section{Discusión}

El presente estudio muestra evidencia de la relación estadísticamente significativa -aunque baja- entre la gaudibilidad y la percepción del estado de salud; resultado que podría ubicarse en la misma dirección de estudios similares en los que algunas variables propias de la psicología positiva correlacionan de manera positiva con aspectos favorables del proceso salud-enfermedad, como se muestra a continuación.

Por ejemplo, en un estudio realizado por Solís-Cámara, Meda-Lara, Moreno-Jiménez, Palomera-Chávez y JuárezRodríguez (2017) con 994 participantes mexicanos de entre 14 y 63 años, se identificó un prototipo de personalidad resiliente caracterizado por un bajo neuroticismo y un alta extraversión, apertura, amabilidad y responsabilidad; rasgos de personalidad característicos de una persona con alta gaudibilidad (Padrós, 2002).

Asimismo, otra variable de la psicología positiva que tiene un peso positivo en relación con la percepción del estado de salud es la de felicidad, incluso en personas con enfermedades crónicas (Vinaccia, Quiceno, Lozano \& Romero, 2017). Frente a esto, al margen de la necesaria clarificación conceptual, las variables de felicidad y gaudibilidad comparten elementos conductuales (Padrós \& Fernández-Castro, 2008), lo que daría a entender que los resultados de este trabajo y los relacionados con el estudio de la felicidad son relevantes en el marco de la búsqueda de factores que mejoran la calidad de las personas, ya sea en población clínica o general.

Sin embargo, es necesario señalar que no todas las variables dentro del marco de la psicología positiva tienen una relación positiva con la percepción del estado de salud. Por ejemplo, en un estudio realizado por Rojas y Ramos (2013) con trabajadores - profesionales y no profesionalesno se identificó correlación entre la percepción del estado de salud y el optimismo disposicional; al igual que en el trabajo realizado por Vinaccia, Quiceno, Medina, Castañeda y Castelblanco (2014), en el cual la resiliencia no tuvo una correlación significativa con la calidad de vida relacionada con la salud en pacientes con dispepsia funcional. A partir de esto, se concluye que no todas las variables de esta perspectiva se relacionan de manera positiva con la percepción del estado de salud, pero sí la gaudibilidad, tal como se mostró en los resultados del presente estudio.

En cuanto a los resultados del Cuestionario de Salud $S F-36$, en particular, se encontraron puntajes medios en todos los factores (medias entre 50 y 60 puntos), y esto contrasta con los reportado por Zúñiga et al. (1999), en donde se encontraron puntajes altos en los factores de $s a$ lud general $(M=72.9, D E=15.5)$ y vitalidad $(M=81.0$, $D E=11.6)$ en una población del sureste de México. Las diferencias entre los resultados de estos estudios pueden ser explicadas por diversos factores, pero, entre los que se consideran más relevantes, se sugiere el incremento de población obesa y el estilo de vida actual. No obstante, deben realizarse futuras investigaciones para evaluar si el puntaje de gaudibilidad es diferente según la región del país y, en ese sentido, si influye diferencialmente en la percepción del estado de salud de las personas.

Por otra parte, a pesar de que en estudios como el de Ware y Sherbourne (1992) se puede apreciar que el Cuestionario de Salud SF-36 discrimina entre población normal y clínica, en un estudio realizado por MartínezHernández et al. (2010) con personas que padecen asma y rinitis se encontró que los participantes obtuvieron medias más bajas en todos los factores de la escala, dato que difiere con lo que se reporta en el presente estudio, como en el factor vitalidad-relacionado con una percepción de energía frente al cansancio y desánimo-, donde la diferencia fue la menor, con siete puntos en la media. Precisamente, esta es una situación que puede explicar la gaudibilidad por ser una variable moduladora relacionada con la percepción del estado de salud, ya que puede influir en el afrontamiento favorable de las personas con alguna enfermedad. Cabe mencionar que vitalidad fue el factor que tuvo el alfa de Cronbach más baja en el presente estudio, en contraste con lo reportado por Martínez-Hernández et al. (2010), por lo cual los resultados deben tomarse con cautela.

Adicionalmente, los resultados de este trabajo muestran que no hay diferencias estadísticamente significativas en la media de la gaudibilidad según el género de los participantes, lo cual contrasta con estudios similares que señalan que el género influye en la capacidad de disfrute (Ruseski, Humphreys, Hallman, Mimbre, Breuer \& 2014). Sin embargo, al igual que en el presente trabajo -pero de forma leve-, diversos estudios señalan que las mujeres perciben mayores problemas de salud tanto en lo físico como en lo psicológico (Solís-Cámara et al., 2017; Uribe, Valderrama \& Molina, 2007), por lo cual se recomienda que las intervenciones sean diseñadas teniendo en cuenta las diferencias en el género; aunque valdría la pena corroborar que los datos reportados se relacionan efectivamente a un problema de salud, pues también podrían estar relacionados con las diferencias de género en cuanto al nivel de autoconocimiento o de respuestas.

Una de las limitaciones del presente estudio fue la transversalidad de la medición, ante lo cual se recomienda 
que futuros estudios evalúen el comportamiento de la gaudibilidad y la percepción del estado de salud en trabajos longitudinales. Además, es necesario realizar estudios con población infantil, ya que podrían dar información importante con respecto a aspectos de la gaudibilidad según el grupo etario.

Finalmente, con base en lo anterior es posible señalar que la gaudibilidad se puede sumar al grupo de variables de la psicología positiva que se relacionan con la percepción del estado de salud, por lo que resulta necesario que se realicen intervenciones en gaudibilidad que permitan identificar la factibilidad de su entrenamiento y sus efectos en la percepción del estado de salud. El campo de investigación de la gaudibilidad es factible.

\section{Referencias}

Instituto Nacional de Estadística y Geografía. (2018). Esperanza de vida. Recuperado de http://cuentame.inegi.org.mx/poblacion/esperanza.aspx?tema $=\mathrm{P}$

Jose, P. E., Lim, B. T., \& Bryant, F. B. (2012). Does savoring increase happiness? A daily diary study. The Journal of Positive Psychology, 7(3), 176-187. doi: 10.1080/17439760.2012.671345

Koenen, K. C., Stellman, S. D., Sommer, J. F., \& Stellman, J. M. (2008). Persisting posttraumatic stress disorder symptoms and their relationship to functioning in Vietnam veterans: a 14-year follow-up. Journal of Traumatic Stress, 21(1), 49-57. doi: 10.1002/jts.20304

Macy, A. S., Theo, J. N., Kaufmann, S. C. V., Ghazzaoui, R. B., Pawlowski, P. A. ... IsHak, W. W. (2013). Quality of life in obsessive compulsive disorder. CNS Spectrums, 18(1), 2133. doi:10.1017/S1092852912000697

Martínez-Hernández, L. E., Segura-Méndez, N. H., AntonioOcampo, A., Torres-Salazar, A., \& Murillo-Gómez, E. (2010). Validación del cuestionario SF-36 en pacientes adultos con asma y rinitis alérgica. Revista Médica Del Instituto Mexicano Del Seguro Social, 48(5), 531-534. Recuperado de http://www.medigraphic.com/pdfs/imss/im2010/im105j.pdf

Mimiaga, M. J., Reisner, S. L., Pantalone, D. W., O’Cleirigh, C., Mayer, K. H., \& Safren, S. A. (2012). A pilot trial of integrated behavioral activation and sexual risk reduction counseling for HIV-uninfected men who have sex with men abusing crystal methamphetamine. AIDS Patient Care STDS, 26(11), 681-693. doi: 10.1089/apc.2012.0216

Montgomery, W. (2009). Mindfulness y gaudibilidad: Categorías en terapia de conducta para tratar la evitación experiencial. Revista de Psicología de la Universidad Inca Garcilaso de la Vega, 1(1), 55-61.

Padrós, F. (2002). Disfrute y bienestar subjetivo. Un estudio psicométrico de la gaudibilidad (tesis de doctorado).
Universidad Autónoma de Barcelona, Barcelona, España. Recuperado de https://ddd.uab.cat/pub/tesis/2002/tdx0602103-202656/tdx.html.orig

Padrós, F., \& Fernández, J. (2001). Escala de Gaudibilidad de Padrós. Una propuesta para medir la disposición a experimentar bienestar. Boletín de Psicología, 71, 7-28. Recuperado de https://www.uv.es/seoane/boletin/previos/ index.html

Padrós, F., \& Fernández-Castro J. A. (2008). A proposal to measure a modulator of the experience of enjoyment: The Gaudiebility Scale. International Journal of Psychology and Psychological Therapy, 8(3), 413-430.

Padrós-Blázquez, F., Herrera-Guzmán, I., \& Gudayol-Ferré, E. (2012). Propiedades psicométricas de la Escala de Gaudibilidad en una población mexicana. Evaluar, 12(1), $1-20$.

Padrós, F., Martínez-Medina, P., \& Graff,A. (2014). Gaudiebility Group Therapy in depressed patients: a pilot study. International Journal of Psychology and Psychological Therapy, 14(1), 59-69. Recuperado de https://www.ijpsy. com/volumen14/num1/374.html

Pickering, A. D., \& Corr, P. J. (2008). J. A. Gray's reinforcement sensitivity theory (RST) of personality. En G. Boyle, G. Matthews \& D. Saklofske (Eds), The SAGE Handbook of Personality: Theory and Assessment Personality Measurement and Testing (Vol. 2) (pp. 239-255). London, New Delhi \& Thousand Oaks: Sage.

Pickering, A. D., Corr, P. J., \& Gray, J. A. (1999). Interactions and reinforcement sensitivity theory: A theoretical analysis of Rusting and Larsen (1997). Personality and Individual Differences, 26(2), 357-365. doi: 10.1016/ S0191-8869(98)00019-1

Rojas, M., \& Ramos, N. (2013). Salud, optimismo y afrontamiento en trabajadores profesionales y no profesionales que trabajan en contextos altamente estresantes. Acta Colombiana de Psicología, 16(1), 149-157. Recuperado de https://editorial.ucatolica.edu.co/ojsucatolica/revistas ucatolica/index.php/acta-colombiana-psicologia/article/ view/256

Ruseski, J. E., Humphreys, B. R., Hallman, K., Mimbre, P., \& Breuer, C. (2014). Sport Participation and Subjective WellBeing: Instrumental Variable Results from German Survey Data. Journal of Physical Activity and Health, 11(2), 396403. doi: 10.1123/jpah.2012-0001

Sánchez-Teruel, D., \& Robles-Bello, M. A. (2017). La gaudibilidad y el bienestar subjetivo en la salud: una revisión sistemática. Revista Latinoamericana de Psicología Positiva, 3(1), 11-23.

Solís-Cámara, Meda-Lara, Moreno-Jiménez, Palomera-Chávez \& Juárez-Rodríguez (2017). Comparación de la salud subjetiva entre prototipos de personalidad recuperados en población general de México. Acta Colombiana de Psicología, 20(2), 200-213. doi:10.14718/ACP.2017.20.2.10 
Sprangers, M. A. G., Bartels, M., Veenhoven, R., Baas, F., Martin, N. G. ... The GENEQOL Consortium (2010). Which patient will feel down, which will be happy? The need to study the genetic disposition of emotional states. Quality of Life Research, 19(10), 1429-1437. doi: 10.1007/ s11136-010- 9652-2

Uribe, A. F., Valderrama, L. J., \& Molina, J. M. (2007). Salud objetiva y salud psíquica en adultos mayores colombianos. Acta Colombiana de Psicología, 10(1), 75-81. Recuperado de https://editorial.ucatolica.edu.co/ojsucatolica/revistas ucatolica/index.php/acta-colombiana-psicologia/article/ view/238

Veenhoven, R.(2003).Artsofliving.JournalofHappiness Studies, 4(4), 373-384. doi: 10.1023/B:JOHS.0000005773.08898.ae

Vinaccia, S., Quiceno, J. M., Lozano, F., \& Romero, S. (2017). Calidad de vida relacionada con la salud, percepción de enfermedad, felicidad y emociones negativas en pacientes con diagnóstico de artritis reumatoide. Acta Colombiana de Psicología, 20(1), 49-59. doi: 10.14718/ACP.2017.20.1.3

Vinaccia, S., Quiceno, J. M., Medina, A., Castañeda, B., \& Castelblanco, P. (2014). Calidad de vida, resiliencia, percepción y conducta de enfermedad en pacientes con diagnóstico de dispepsia funcional. Acta Colombiana de Psicología, 17(1), 61-70. doi: 10.14718/ACP.2014.17.1.7

Ware, J. E., \& Sherbourne, C. D. (1992). The MOS 36-item Short Form Health Survey (SF-36): I. Conceptual framework and item selection. Medical Care, 30(6), 473-483. doi: 10.1007/BF03260127

Zúñiga, M. A., Carrillo-Jiménez, G. T., Fos, P. J., Gandek, B., \& Medina-Moreno, M. R. (1999). Evaluación del estado de salud con la Encuesta SF-36: resultados preliminares en México. Salud Pública de México, 41(2), 110-118. doi: 10.1590/S0036-36341999000200005 